\title{
Expression and tissue distribution of human X-linked armadillo repeat containing-6
}

\author{
YUSUKE KUSAMA, SAYURI TAKAYANAGI, MOE TATEGU and KENICHI YOSHIDA \\ Department of Life Sciences, Faculty of Agriculture, Meiji University, Kanagawa 214-8571, Japan
}

Received December 11, 2009; Accepted January 29, 2010

DOI: $10.3892 /$ etm_00000062

\begin{abstract}
The armadillo family of proteins has been implicated in embryogenesis and tumorigenesis. Armadillo repeat containing X-linked (ARMCX)1-6 and its most closely related protein, ARMC10, share an uncharacterized domain in their carboxyl-terminal region and thereby constitute a unique subfamily. We previously detected an elevated ARMCX6 mRNA level in human cervical carcinoma (HeLa) cells in which the cyclin-dependent kinase inhibitor 2A products (p16INK4A and p14ARF) were silenced by RNA interference; however, the function of the $A R M C X 6$ gene has yet to be determined. In the present study, we demonstrated that ARMCX6 tagged with green fluorescent protein was localized in the cytoplasm of HeLa cells. ARMCX6 mRNA was highly detected in the pancreas and spleen, indicating a tissuespecific expression pattern in human tissues. Taken together, these results suggest that $A R M C X 6$ may be uniquely involved in a specific cellular function of certain tissues.
\end{abstract}

\section{Introduction}

The armadillo family of proteins is growing rapidly in number. This family exhibits diverse functions, such as embryogenesis and tumorigenesis, by interacting with multiple binding partners at their characteristic armadillo repeat (1). Among these proteins, $\beta$-catenin has been intensively investigated as a crucial integrator of the Wnt signaling pathway as well as cell-cell adhesions (2). To date, a unique subfamily whose chromosomal localization

Correspondence to: Dr Kenichi Yoshida, Department of Life Sciences, Faculty of Agriculture, Meiji University, 1-1-1 Higashimita, Tama-ku, Kawasaki, Kanagawa 214-8571, Japan

E-mail: yoshida@isc.meiji.ac.jp

Abbreviations: aa, amino acid; ARMCX, armadillo repeat containing, X-linked; GAPDH, glyceraldehyde-3-phosphate dehydrogenase; GFP, green fluorescent protein; PCR, polymerase chain reaction; RT, reverse transcription

Key words: armadillo repeat containing, X-linked, gene expression, human tissues has been identified as chromosome region $\mathrm{Xq}$ has been discovered; this subfamily is referred to as the armadillo repeat containing, X-linked (ARMCX) subfamily. ARMCX1, $A R M C X 2$ and $A R M C X 3$, also known as ALEX1, ALEX2 and $A L E X 3$, respectively, are located at the chromosome region Xq21.33-q22.2 and have been implicated in tumorigenesis (3). Their amino $(\mathrm{N})$-terminal region harbors a transmembrane domain, suggesting that these proteins may localize at membrane structures in cells. Recently, ARMCX3 was revealed as an integral membrane protein of the mitochondrial outer membrane that functionally interacts with the transcriptional regulator Sox10 (4). In addition, ARMCX4, ARMCX5 and ARMCX6, the localizations of which have been mapped to chromosome regions Xq22.1, Xq22.1q22.3 and Xq21.33-q22.3, respectively, were also identified. Among them, ARMCX5 was found to be activated and bound by oncogene ZNF217 (5). An oligonucleotide array identified ARMCX6 as having an at least 2-fold increase in mRNA expression in peripheral blood mononuclear cells from patients with rheumatoid arthritis when compared with control subjects (6). We previously identified ARMCX6 as an up-regulated gene in both p16INK4A- and p14ARF-silenced HeLa cells (7). Both p16INK4A and p14ARF were found to be frequently deleted or inactivated in various types of cancer (8).

In the present study, we revealed that $A R M C X 6$ was exclusively localized in the cytoplasm of HeLa cells. Tissue distribution of ARMCX6 was highly detected in the pancreas and spleen. Taken together, these findings suggest that ARMCX6 may be useful as a diagnostic marker for the carcinogenesis of specific cells or tissues.

\section{Materials and methods}

Bioinformatics. Pfam and PROSITE (pattern/profile) (http:// motif.genome.jp/), CLUSTAL W (ver. 1.81) Multiple Sequence Alignments (http://align.genome.jp/), SOSUI (ver. 1.11) (http:// bp.nuap.nagoya-u.ac.jp/sosui/) and PSORT II and iPSORT (http://psort.ims.u-tokyo.ac.jp/) predictions were performed using web-based free software. The GenBank accession numbers that were used were ARMCX1 (NM_016608), ARMCX2 (NM_014782), ARMCX3 (NM_016607), ARMCX4 (AK292543), ARMCX5 (NM_022838), ARMCX6 (NM_019007), armadillo repeat containing 10 (ARMC10) (alias SVH) (NM_031905), G-protein coupled receptor- 
associated sorting protein 1 (GPRASPI) (NM_014710) and GPRASP2 (NM_001004051).

Cell cultures, plasmid, transfection and fluorescent microscopy. Human cervical carcinoma (HeLa) cells were cultured in Earle's modified Eagle's medium (MEM) supplemented with $10 \%$ fetal bovine serum, $1 \%$ non-essential amino acids and antibiotic-antimycotics (all from Invitrogen, Carlsbad, CA, USA). The ARMCX6 EST (expressed sequence tag) cDNA clone (GenBank no. BC007677; IMAGE 3609980; Open Biosystems, Huntsville, AL, USA) was used as a template to amplify the $A R M C X 6$-coding region using a polymerase chain reaction (PCR), and the coding region was ligated into the respective enzyme EcoRI and BamHI sites of the pEGFP-N2 vector (Clontech, Mountain View, CA, USA). The PCR primer set was as follows (underlines indicate the flanking enzyme site): CGGAATTCGCCACCATGGGCCGGGCTCGGGAAGTG and CGGGATCCATGGGGCAGGGGTTTCCAG. The plasmid sequence was verified by sequencing at the Takara facility (Mie, Japan). The cells were transfected using Lipofectamine Plus (Invitrogen) or the Neon Transfection System (Invitrogen) according to the manufacturer's instructions. Briefly, Neon electroporation was performed in a 24-well plate with a pulse voltage of 1,200 and a pulse width of 40. After 24-48 $\mathrm{h}$ of transfection, the cells were washed using phosphate-buffered saline (PBS) and photographed using a fluorescent microscope DM IRB (Leica, Wetzlar, Germany), equipped with a digital single-lens reflex camera (Camedia E-20; Olympus, Tokyo, Japan).

Reverse transcription (RT)-PCR. The total RNA samples were prepared using an RNeasy Mini-Spin Column (Qiagen, Valencia, CA, USA) in accordance with the manufacturer's instructions. Prior to RT-PCR, total RNA was treated with deoxyribonuclease I (Invitrogen) according to the manufacturer's instructions. One or two micrograms of total RNA was reverse transcribed using High-Capacity cDNA Reverse Transcription Kits (Applied Biosystems, Foster City, CA, USA). The PCR was carried out in $25 \mu \mathrm{l}$ of a mix consisting of $1 \mathrm{X}$ buffer, $200 \mu \mathrm{M}$ of dNTPs, $400 \mathrm{nM}$ of primers, $1 \mathrm{mM}$ of $\mathrm{MgSO}_{4}, 5 \%$ DMSO and 1 unit of KOD plus DNA polymerase (Toyobo, Osaka, Japan). Hot-start PCR was then performed as follows: denaturation for $3 \mathrm{~min}$ at $94^{\circ} \mathrm{C}$, followed by ad libitum cycles at $94^{\circ} \mathrm{C}$ for $15 \mathrm{sec}$, a gene-specific annealing temperature $\left[A R M C X 3,56^{\circ} \mathrm{C} ; A R M C X 4,56^{\circ} \mathrm{C} ; A R M C X 5,57^{\circ} \mathrm{C}\right.$; $A R M C X 6,54^{\circ} \mathrm{C} ; A R M C 10,67^{\circ} \mathrm{C}$; glyceraldehyde-3-phosphate dehydrogenase $\left.(G A P D H), 60^{\circ} \mathrm{C}\right]$ for $30 \mathrm{sec}$ and $68^{\circ} \mathrm{C}$ for $30 \mathrm{sec}$, followed by an extension step of $3 \mathrm{~min}$ at $68^{\circ} \mathrm{C}$. The PCR results were verified by varying the number of PCR cycles for each cDNA and set of primers. The target gene primer pairs were as follows: ARMCX3, GGGCTGTCCAGA AACGGGCT and CCCTGAGCAGTTCCCTAGTC for $540 \mathrm{bp}$; ARMCX4, GCAAGAAGTGGGCCTAGGGC and TTCCCT GGGTACTGCCAAGG for $420 \mathrm{bp}$; ARMCX5, GGCCTAAT CCGAAGGCCTGC and CCAGCTGCACAGGGGAGTTC for $360 \mathrm{bp}$; ARMCX6, GGGCTCAATCCAGGACCACA and GTTCACTATCCATCAGGCGC for $350 \mathrm{bp}$; ARMC10, TCAC CTGCCAACCTGACCAT and GCGCTATCTCAGCTCAC TGC for $460 \mathrm{bp}$; and GAPDH (NM_002046), CCATGGC AAATTCCATGGCA and GTCCTTCCACGATACCAAAG for $365 \mathrm{bp}$. The amplified products were separated on $1.0 \%$ agarose gels and visualized under ultraviolet transillumination. For the cDNA panel analysis, $2.5 \mu \mathrm{l}$ of cDNA purchased from Clontech was used (Human MTC panels I and II cDNA panel). The GAPDH primer in the kit was used as a control to amplify $983 \mathrm{bp}$.

Western blotting. The cells were harvested and lysed in modified RIPA lysis buffer [50 mM Tris- $\mathrm{HCl}(\mathrm{pH} 7.5)$, $150 \mathrm{mM} \mathrm{NaCl}, 1 \%$ Nonidet P40, $0.5 \%$ sodium deoxycholate and $1 \mathrm{mM}$ EDTA] containing a protease inhibitor cocktail (Sigma, St. Louis, MO, USA) for $20 \mathrm{~min}$ on ice. The cell lysates were centrifuged, and the protein concentrations were determined using the Bio-Rad Protein Assay Kit (Bio-Rad Laboratories, Hercules, CA, USA). The protein lysates were loaded onto each lane of a gel. Before performing sodium dodecyl sulfate polyacrylamide gel electrophoresis (SDS-PAGE), the reaction was terminated by the addition of Laemmli sample buffer containing $100 \mathrm{mM}$ of dithiothreitol (DTT). Equal amounts of cellular protein were electrophoresed on NuPAGE 4-12\% Bis-Tris gel with MES running buffer (Invitrogen) and transferred to a HybondPVDF membrane (GE Healthcare, Piscataway, NJ, USA). The membrane was first blocked using PBS containing 0.1\% Tween-20 and 5\% non-fat dried milk, then incubated with GFP (JL-8; Clontech) and GAPDH (Applied Biosystems) antibodies. Alkaline phosphatase (AP)-labeled secondary antibodies were purchased from Promega (Madison, WI, USA). A Western Blue-stabilized substrate was used to detect the signals, according to the manufacturer's protocol (Promega).

\section{Results}

Characteristic features of ARMCXs. To determine the functional aspects of ARMCX6, we predicted the motifs or domains of the amino acids (aa) composing ARMCX6 using a Pfam and PROSITE (pattern and profile) search. Pfam revealed that a protein of unknown function (referred to as DUF634) is a characteristic feature of the carboxyl (C)-terminal of the ARMCX6 protein (9-300 aa of full-length 300 aa). In addition to ARMCX6, DUF634 was observed in other proteins including ARMCX1 (184-447 aa of full-length 453 aa), ARMCX2 (365-626 aa of full-length 632 aa), ARMCX3 (100-363 aa of full-length 379 aa), ARMCX5 (288-552 aa of full-length 558 aa) and ARMC10 (74-337 aa of full-length 343 aa). Among the ARMCXs, DUF634 was not identified in ARMCX4. DUF634 was also recognized in the C-terminus of GPRASP1 (1,132-1,384 aa of full-length 1,395 aa) and GPRASP2 (574-836 aa of full-length 838 aa). In contrast, Arm (Armadillo/ $\beta$-catenin-like repeat) was exclusively predicted in ARMCX1 (239-276 aa), ARMCX2 (419-457 aa), ARMCX3 (155-192 aa) and ARMC10 (127-167 aa). Based on PROSITE, ARM_REPEAT (Armadillo/plakoglobin ARM repeat motif) was only noted in ARMCX1 (247-284 aa) and ARMCX3 (163-200 aa).

Next, we performed CLUSTAL W multiple sequence alignments using full-length ARMCX1 (453 aa), ARMCX2 (632 aa), ARMCX3 (379 aa), ARMCX4 (348 aa), ARMCX5 (558 aa), ARMCX6 (300 aa) and ARMC10 (343 aa). 
Table I. PSORT II prediction of subcellular localization of ARMCXs.

\begin{tabular}{llllll}
\hline ARMCX1 & ARMCX2 & ARMCX3 & ARMCX4 & ARMCX5 & \multirow{2}{*}{ ARMCX6 } \\
\hline Mit (39.1) & Cyt (39.1) & Mit (34.8) & Cyt (69.6) & Nuclear (56.5) & Ext (44.4) \\
Cyt (21.7) & Mit (26.1) & Cyt (26.1) & Nuclear (17.4) & Mit (30.4) & Cyt (22.2) \\
ER (17.4) & Nuclear (17.4) & Vacuolar (8.7) & Mit (8.7) & Cyt (8.7) & Vacuolar (11.1) \\
Golgi (8.7) & Vacuolar (8.7) & Ext (8.7) & Per (4.3) & Cytoskeletal (4.3) & Nuclear (11.1) \\
Nuclear (8.7) & Per (4.3) & Nuclear (8.7) & & & ER (11.1) \\
Vacuolar (4.3) & ER (4.3) & ER (8.7) & & & \\
& & Golgi (4.3) & & & \\
\hline
\end{tabular}

Mit, mitochondrial; Cyt, cytoplasmic; ER, endoplasmic reticulum; Per, peroxisomal; Ext, extracellular including cell wall. The numbers in the parentheses indicate the percentage $(\%)$ of the predicted subcellular localization.

GPRASP1 and GPRASP2 apparently had longer coding regions compared to ARMCXs and ARMC10; therefore, these two proteins were excluded from further analysis. Consistent with the DUF634 domain, the C-terminals of each of the proteins were similar (data not shown), suggesting the evolutionarily conserved functional importance of this region. Previously, ARMCX1, ARMCX2 and ARMCX3 were predicted to possess similar $\mathrm{N}$-terminal transmembrane domain and armadillo repeats (3). Based on the alignment of ARMCX1-6 and ARMC10, conserved amino acids were detected in the $\mathrm{N}$-terminal transmembrane domain (data not shown); however, SOSUI prediction revealed that one transmembrane helix was identified in the N-terminal of ARMCX1 (5-27 aa), ARMCX2 (5-27 aa) and ARMCX3 (7-29 aa) as well as the central region of ARMCX4 (96-118 aa) but not in any region of ARMCX5 or ARMCX6. SOSUI also predicted that ARMCX5 and ARMCX6 may be soluble proteins. On the other hand, iPSORT predicted that the N-terminal (1-30 aa) of ARMCX6 as well as ARMCX1-3 and ARMC10 may be a signal peptide. Apparently, ARMCX4 and ARMCX5 do not possess a signal peptide.

To predict the subcellular localization of ARMCXs, PSORT II prediction was performed. ARMCX6 had a $44.4 \%$ probability of localizing in an extracellular compartment, including the cell wall, whereas the other ARMCXs did not have a high probability of being localized extracellularly (Table I). Based on published information, PSORT II prediction is a reliable bioinformatics tool. Indeed, ARMCX3 has been shown to be an integral membrane protein of the mitochondrial outer membrane (4). In addition to the ARMCXs, ARMC10 also possesses a transmembrane domain and has been shown to localize in the endoplasmic reticulum (10). PSORT II predicted that ARMC10 is localized in vacuolar regions $(22.2 \%)$, extracellular regions including the cell wall (22.2\%), the Golgi apparatus (22.2\%), mitochondrial regions $(22.2 \%)$ and the endoplasmic reticulum (11.1\%). These cellular regions are functionally proximal to the endoplasmic reticulum, as they all are membrane-enclosed organelles. To determine the cellular localization of ARMCX6, we constructed a C-terminal green fluorescent protein (GFP)-tagged ARMCX6 expression plasmid. Fluorescent microscopy revealed a few GFP-positive cells in a GFP-tagged ARMCX6-transfected well, whereas abundant GFP-positive
A

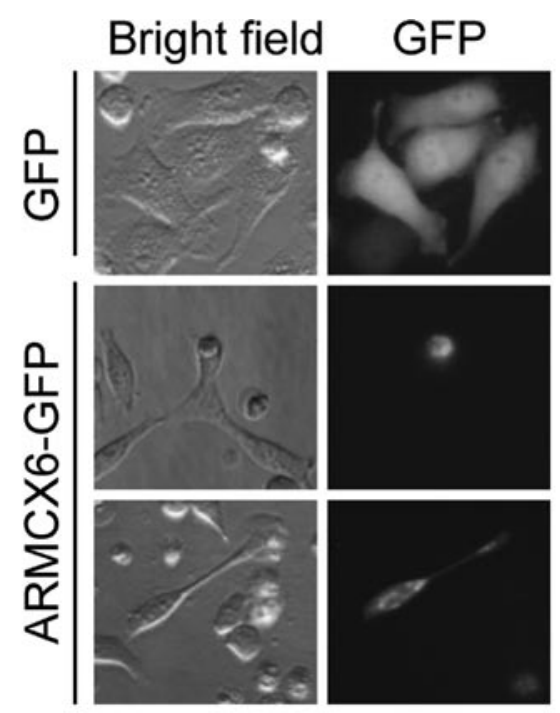

B

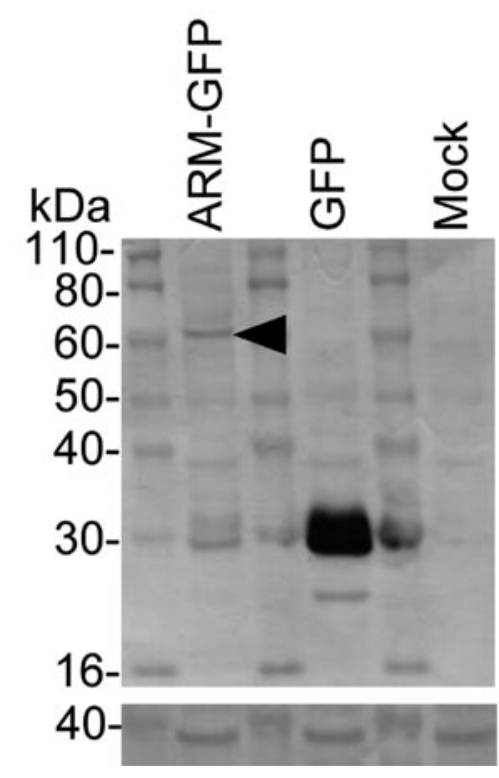

Figure 1. Expression of ARMCX6 in HeLa cells. (A) Fluorescence microscope detection of GFP (upper/right panel) and ARMCX6-GFP- (middle/ right and lower/right panel) transfected HeLa cells. A bright field is also presented (left panel). All images were captured at 400-fold magnification. (B) Western blot analysis of ARMCX6 protein fused to GFP. Anti-GFP detection of ARMCX6-GFP, GFP and Mock-transfected HeLa cells. The arrowhead indicates the position of ARMCX6-GFP (upper panel). GAPDH was shown as a loading control (lower panel). The positions of the molecular weight markers $(\mathrm{kDa})$ are shown on the left side of the panel. 
B
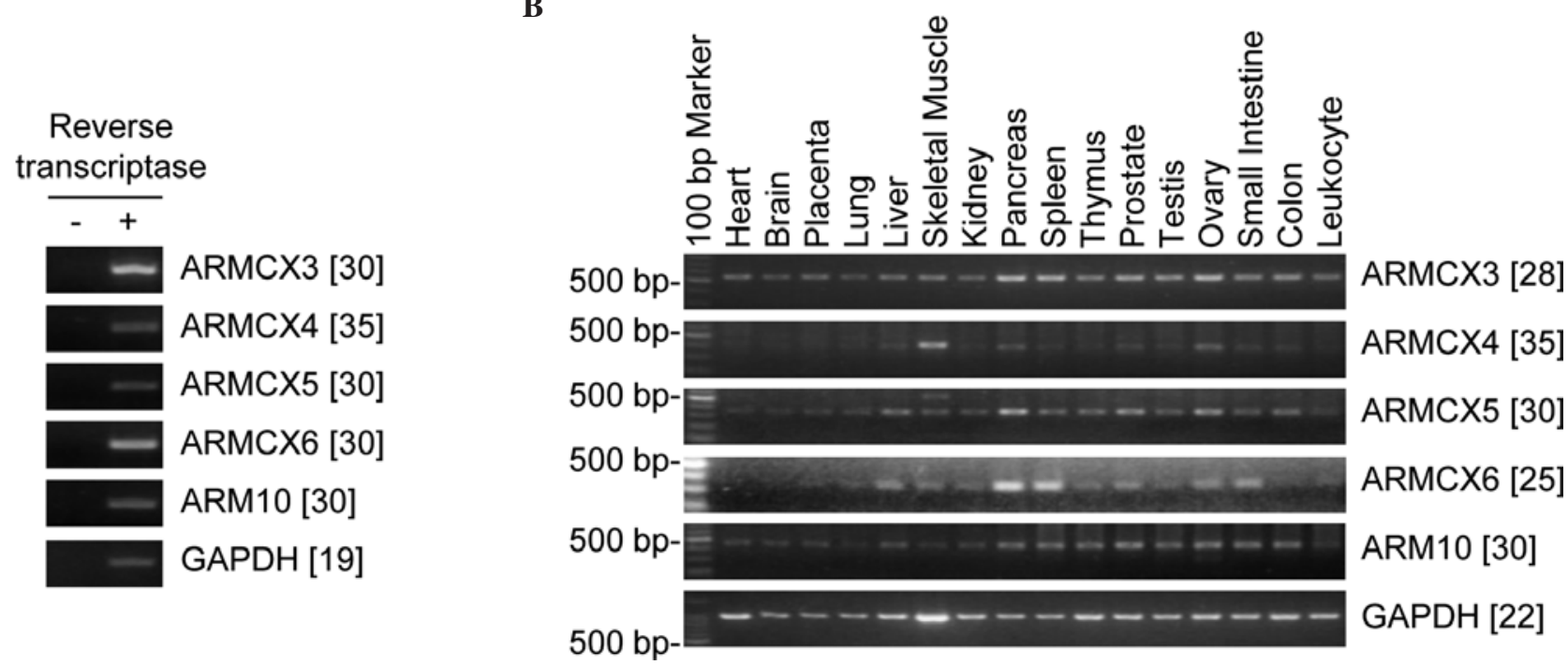

Figure 2. Comparative tissue distribution patterns of ARMCXs. (A) RT-PCR detection of ARMCX3-6 and ARMC10 with or without reverse transcriptase in HeLa cells. The PCR cycle numbers for cDNA amplification are indicated in square brackets. (B) Tissue distribution of ARMCX3-6 and ARMC10 mRNA. PCR detection of ARMCX3-6 and ARMC10 cDNA derived from human adult tissues. The position of the 500-bp band is indicated. The square brackets indicate the PCR cycle numbers.

cells were detected in a control GFP-transfected well (data not shown). In the GFP-tagged ARMCX6-transfected well, the GFP-positive cells were all round in shape and had shrunken, but the control GFP-transfected cells showed a typical extended shape (Fig. 1A, middle panel vs. upper panel). Exceptionally, GFP-positive intact cells were detected in the GFP-tagged ARMCX6-transfected well. In it, GFP-tagged ARMCX6 was diffusely spread throughout the cells, with the probable exception of the nuclei (Fig. 1A, lower panel).

After $48 \mathrm{~h}$ of transfection, using an anti-GFP antibody, an ARMCX6-GFP fusion protein was detected as a faint band with an estimated molecular weight of $\sim 60 \mathrm{kDa}$, compared with the abundantly expressed 27-kDa GFP (Fig. 1B, upper panel). GAPDH was consistently detected in all lanes (Fig. 1B, lower panel). The ARMCX6 protein seems to be unstable, since another expression system FLAG-tagged ARMCX6 again produced a faint band (data not shown). Furthermore, we constructed N-terminal deleted FLAG-tagged ARMCX6 constructs to exclude the signal peptide but failed to detect a reasonable protein level using immunoblotting (data not shown). Taken together, these results suggest that ARMCX6 is not a secreted protein and cellular toxicity is induced by ectopic ARMCX6 expression.

Comparative expression analysis of ARMCXs. Next, we focused on the transcriptional regulation of ARMCXs, the chromosomal localizations of which are uniquely mapped to the same region of Xq. ARMC10 is localized at 7q22.1 but was included in this investigation. To date, the mRNA distributions of ARMCXI and ARMCX2 in human tissues have been reported (3), therefore ARMCX1 and ARMCX2 were omitted from the present study. The primer set used to detect ARMCX3-6 and ARMC10 was able to specifically amplify their mRNAs in the case of RT plus- but not RT minus-derived cDNAs (Fig. 2A). The expression levels of
ARMCX3-6 and ARMC10 were examined using a multiple tissue-derived cDNA pool that included 16 adult tissues. ARMCX6 mRNA was predominantly detected in the pancreas and spleen, moderately detected in the liver, skeletal muscle, kidney, thymus, prostate, testis, ovary and small intestine, and scarcely detected in the placenta and leukocytes; ARMCX6 mRNA was not detected in the heart, brain, or colon (Fig. 2B). In contrast to $A R M C X 6, A R M C X 3-5$ and $A R M C 10$ showed a relatively ubiquitous expression pattern (Fig. 2B).

\section{Discussion}

A unique aspect of the $A R M C X$ subfamily of armadillo family proteins is their chromosomal localization, which is mapped to Xq, and their presumed involvement in tumorigenesis $(1,3)$. We previously identified ARMCX6 as an up-regulated gene in p16INK4a and p14ARF knocked down HeLa cells (7). The cyclin-dependent kinase inhibitor $2 \mathrm{~A}(C D K N 2 A)$ gene alternatively produces two protein products, p16INK4A and p14ARF, and both proteins have crucial roles in cell fate determination as upstream regulators of $\mathrm{pRb}$ and $\mathrm{p} 53$, respectively (8). In the present study, we aimed to reveal the functional aspect of ARMCX6 and the expression pattern of ARMCXs.

Based on predictions made using their amino acid compositions, ARMCXs tend to be membrane-associated proteins. Based on sequence homology, ARMCX6 seems to be localized or associated with the membrane structure of cells. We investigated the ARMCX6 subcellular localization using an ARMCX6-GFP expression plasmid. ARMCX6-expressing cells tended to become atrophic compared to control cells, indicating that ARMCX6 may cause cellular toxicity. In addition, the number of ectopically ARMCX6-expressing cells was very low, compared to that of the control GFP-transfected cells. The ARMCX6 protein may have a short half-life. A future functional study examining ARMCX6 is needed. 
The mRNA expression patterns of ARMCXI and $A R M C X 2$ have been reported in various human tissues (3). These patterns were remarkably similar; namely, high expression levels were observed in the ovary, heart, testis, prostate, brain, spleen and colon; faint expression levels were observed in the liver and thymus; and no expression was observed in leukocytes. In contrast, the ARMCX6 mRNA levels were high in the pancreas and spleen, and no expression was observed in the heart, brain and colon. ARMCX1 and ARMCX2 were originally described as genes that are down-regulated in epithelial cancers (3). In addition, ARMC10 is known to be up-regulated in hepatocellular carcinomas (9). These results support the idea that ARMCX6 may be a novel biomarker of cell fate determination in specific tissues or neoplasms derived from certain tissues.

\section{Acknowledgements}

This work was supported by a Research Project Grant (B) from the Institute of Science and Technology, Meiji University, and by a Grant from the Daiwa Securities Health Foundation. We thank the members of the Yoshida laboratory for the technical assistance.

\section{References}

1. Hatzfeld M: The armadillo family of structural proteins. Int Rev Cytol 186: 179-224, 1999.

2. Brembeck FH, Rosário M and Birchmeier W: Balancing cell adhesion and Wnt signaling, the key role of beta-catenin. Curr Opin Genet Dev 16: 51-59, 2005.

3. Kurochkin IV, Yonemitsu N, Funahashi SI and Nomura H: ALEX1, a novel human armadillo repeat protein that is expressed differentially in normal tissues and carcinomas. Biochem Biophys Res Commun 280: 340-347, 2001.

4. Mou Z, Tapper AR and Gardner PD: The armadillo repeat-containing protein, ARMCX3, physically and functionally interacts with the developmental regulatory factor Sox10. J Biol Chem 284: 13629-13640, 2009.

5. Krig SR, Jin VX, Bieda MC, O'Geen H, Yaswen P, Green R and Farnham PJ: Identification of genes directly regulated by the oncogene ZNF217 using chromatin immunoprecipitation (ChIP)-chip assays. J Biol Chem 282: 9703-9712, 2007.

6. Edwards CJ, Feldman JL, Beech J, Shields KM, Stover JA, Trepicchio WL, Larsen G, Foxwell BM, Brennan FM, Feldmann M and Pittman DD: Molecular profile of peripheral blood mononuclear cells from patients with rheumatoid arthritis. Mol Med 13: 40-58, 2007.

7. Sato K, Kusama Y, Tategu M and Yoshida K: FBXL16 is a novel E2F1-regulated gene commonly upregulated in p16INK4A- and p14ARF-silenced HeLa cells. Int J Oncol 36: 479-490, 2010.

8. Sharpless NE and DePinho RA: The INK4A/ARF locus and its two gene products. Curr Opin Genet Dev 9: 22-30, 1999.

9. Huang R, Xing Z, Luan Z, Wu T, Wu X and Hu G: A specific splicing variant of $\mathrm{SVH}$, a novel human armadillo repeat protein, is up-regulated in hepatocellular carcinomas. Cancer Res 63: 3775-3782, 2003. 